\title{
Length of the Mitral Isthmus But Not Anatomical Location of Ablation Line Predicts Bidirectional Mitral Isthmus Block in Patients Undergoing Catheter Ablation of Persistent Atrial Fibrillation: A Randomized Controlled Trial
}

\author{
DANIEL SCHERR, M.D.,,$\dagger$ NICOLAS DERVAL, M.D., ${ }^{*}$ MANAV SOHAL, M.B.B.S. $\ddagger$ \\ PATRIZIO PASCALE, M.D., ${ }^{*}$ MATTHEW WRIGHT, M.B.B.S., PH.D., ${ }^{*}, \ddagger$ AMIR JADIDI, M.D., ${ }^{*}$ \\ YUKI KOMATSU, M.D., ${ }^{*}$ LAURENT ROTEN, M.D., ${ }^{*}$ STEPHEN B. WILTON, M.D., ${ }^{*}$ \\ MICHALA PEDERSEN, M.D., ${ }^{*}$ KHALED RAMOUL, M.D., ${ }^{*}$ SHINSUKE MIYAZAKI, M.D., ${ }^{*}$ \\ ASHOK SHAH, M.D., ${ }^{*}$ NICK LINTON, M.D., ${ }^{*}$ MARTIN MANNINGER, M.D., $\dagger$ ARNAUD DENIS, \\ M.D., ${ }^{*}$ MELEZE HOCINI, M.D., ${ }^{*}$ FREDERIC SACHER, M.D., ${ }^{*}$ MICHEL HAISSAGUERRE, M.D., ${ }^{*}$ \\ PIERRE JAIS, M.D., F.H.R.S., ${ }^{*}$ and SEBASTIEN KNECHT, M.D., PH.D.*
}

From the *Hôpital Cardiologique du Haut Lévêque, Université Victor-Segalen Bordeaux, Pessac, France; †Division of Cardiology, Department of Medicine, Medical University of Graz, Austria; and $\ddagger$ Kings College London BHF Centre, Cardiovascular Division, NIHR Biomedical Research Centre at Guy's and St. Thomas' NHS Foundation Trust, London, UK

Importance of Line and Length for Mitral Isthmus. Introduction: Mitral isthmus (MI) ablation is an effective option in patients undergoing ablation for persistent atrial fibrillation (AF). Achieving bidirectional conduction block across the MI is challenging, and predictors of $\mathrm{MI}$ ablation success remain incompletely understood. We sought to determine the impact of anatomical location of the ablation line on the efficacy of MI ablation.

Methods and Results: A total of 40 consecutive patients (87\% male; $54 \pm 10$ years) undergoing stepwise AF ablation were included. MI ablation was performed in sinus rhythm. MI ablation was performed from the left inferior PV to either the posterior (group 1) or the anterolateral (group 2) mitral annulus depending on randomization. The length of the MI line (measured with the 3D mapping system) and the amplitude of the EGMs at 3 positions on the MI were measured in each patient. MI block was achieved in 14/19 (74\%) patients in group 1 and 15/21 (71\%) patients in group $2(P=N S)$. Total MI radiofrequency time $(18 \pm$ 7 min vs. $17 \pm 8$ min; $P=$ NS) was similar between groups. Patients with incomplete MI block had a longer MI length $(34 \pm 6 \mathrm{~mm}$ vs. $24 \pm 5 \mathrm{~mm} ; \mathrm{P}<0.001)$, a higher bipolar voltage along the MI $(1.75 \pm 0.74 \mathrm{mV}$ vs. $1.05 \pm 0.69 \mathrm{mV} ; P<0.01)$, and a longer history of continuous $\mathrm{AF}(19 \pm 17$ months vs. $10 \pm 10$ months; $P<\mathbf{0 . 0 5}$ ). In multivariate analysis, decreased length of the MI was an independent predictor of successful MI block (OR 1.5; 95\% CI 1.1-2.1; P < 0.05).

Conclusions: Increased length but not anatomical location of the MI predicts failure to achieve bidirectional MI block during ablation of persistent AF. (J Cardiovasc Electrophysiol, Vol. 26, pp. 629-634, June 2015)

ablation, atrial fibrillation, mitral isthmus, conduction block, atrial tachycardia

\begin{abstract}
Dr. Daniel Scherr was supported by the ESC/EHRA Fellowship in Clinical Electrophysiology. Matthew Wright and Manav Sohal acknowledge financial support from the Department of Health via the National Institute for Health Research (NIHR) comprehensive Biomedical Research Centre award to Guy's and St. Thomas' NHS Foundation Trust in partnership with King's College London and King's College Hospital NHS Foundation Trust. The views expressed are those of the author and not necessarily those of the NHS, the NIHR, or the Department of Health.
\end{abstract}

S.B. Wilton reports participation on a research grant supported by St. Jude Medical. M. Hocini, M. Haissaguerre, and P. Jais report research support from Biosense Webster, St. Jude Medical, and Boston Scientific. Other authors: No disclosures.

Address for correspondence: Manav Sohal, M.B.B.S., Kings College London BHF Centre, Cardiovascular Division, NIHR Biomedical Research Centre at Guy's and St. Thomas' NHS Foundation Trust. 4th Floor Lambeth Wing, St. Thomas' Hospital, London SE1 7EH, UK. Fax: 22-207-188-5442; E-mail:manav.sohal@gstt.nhs.uk

Manuscript received 26 November 2014; Revised manuscript received 10 February 2015; Accepted for publication 20 February 2015.

\section{Introduction}

Catheter ablation is an established treatment option for patients with symptomatic atrial fibrillation (AF). In paroxysmal AF (PAF) ablation, pulmonary vein isolation (PVI) is a well-defined procedural endpoint. In persistent AF (PsAF), PVI alone results in modest freedom of AF, with mediumterm success rates ranging from $11-46 \%,{ }^{1-4}$ and additional substrate modification is necessary to increase the success rate. Previous studies have shown that linear ablation at the mitral isthmus (MI) in addition to PVI increases the rate of sinus rhythm (SR) maintenance in persistent AF patients. ${ }^{5-7}$

Hypotheses concerning the additional benefit of the MI ablation line during AF ablation are the following: (1) the elimination anatomic or functional reentries (rotors/macroreentrant AT), (2) elimination ofarrhythmogenic

doi: $10.1111 /$ jce. 12667 
triggers (i.e., ligament of Marshall), and (3) impact on autonomic innervation.

Performing the MI line, however, is difficult and acute bidirectional block is achieved in a limited amount of patients. 5,6,8,9 Increased success is often associated with increased risk of complications from higher ablation power and higher risk of steam pop, perforation, and tamponade. ${ }^{10}$ Furthermore, incomplete block across left atrial (LA) ablation lines can be responsible for proarrhythmia and atrial tachycardia (AT) recurrence. ${ }^{6,11,12}$ The 2012 HRS/EHRA/ ECAS AF ablation guidelines state that if additional linear lesions are applied in persistent AF (PsAF) ablation, line completeness should be assessed. ${ }^{13}$ While the importance of the MI line has been demonstrated, the predictors of MI ablation success and preferential anatomical location of the MI line have not been assessed.

The aim of this prospective randomized study therefore was to (1) determine the impact of the exact anatomical location of the ablation line on efficacy of MI ablation in PsAF ablation and (2) identify the predictors of successful MI ablation.

\section{Methods}

\section{Study Population}

The study population comprised 40 consecutive patients undergoing their first catheter ablation for symptomatic and drug-refractory persistent AF between November 2010 and February 2012 at Hopital Cardiologique du Haut-Leveque, Bordeaux-Pessac. The research protocol was approved by the local ethics committee and all patients provided written informed consent. Persistent AF was defined as AF sustaining beyond 7 days, or lasting $<7$ days but necessitating pharmacological or electrical cardioversion; longstanding persistent AF was defined as continuous AF of $>1$-year duration. ${ }^{13}$ All patients gave written informed consent according to the institutional guidelines of the University of Bordeaux Health System (who provided ethical approval for the study). Patients were randomized in 1:1 fashion to either posterior or anterolateral isthmus lines.

\section{Electrophysiological Study}

Antiarrhythmic medications were discontinued $\geq 5$ halflives prior to ablation with the exception of amiodarone. All patients were on coumadin oral anticoagulantion and achieved a target INR of 2-3 for at least 1 month prior to ablation. Transesophageal echocardiography was performed within 5 days of the procedure to exclude atrial thrombus. Oral anticoagulation was stopped 48 hours before the procedure and resumed on the day following the ablation.

Femoral venous access was established under local anesthesia and conscious sedation. A steerable quadripolar or decapolar catheter $(5 \mathrm{~mm}$ electrode spacing, Xtrem, ELA Medical, Montrouge, France) was placed within the CS. Following transseptal access, an intravenous bolus of heparin $(0.5 \mathrm{mg} / \mathrm{kg}$ of body weight) was administered and repeated when the activated clotting time was $<250$ seconds.

Surface electrocardiograms (ECGs) and intracardiac electrograms (EGMs) were continuously monitored at a sweep speed of $100 \mathrm{~mm} / \mathrm{s}$ and recorded (Labsystem Pro, Bard, Tewksbury, MA, USA) and were filtered from 0.05 to $100 \mathrm{~Hz}$ and 30 to $500 \mathrm{~Hz}$, respectively.

\section{Mitral Isthmus Line Characteristics and Ablation}

A 3D anatomical map of the left atrium was made at the outset of the case using Carto $3^{\mathrm{TM}}$ (Biosense Webster, Diamond Bar, CA, USA) with careful attention paid to identification of the PVs as well as the mitral annulus (Fig. 1). The area subtended by either MI line was segmented on the map so that no ablation would be directed to this area during pulmonary vein isolation and ablation of complex fractionated electrograms (CFAEs).

Following pulmonary vein isolation, CFAE ablation and linear lesion at the roof and the cavotricuspid isthmus (CTI) were performed. If the patient was still in AF or tachycardia, DC cardioversion was performed to achieve sinus rhythm. MI measurements and linear ablation were always carried out during sinus rhythm. The MI line was performed during CS pacing (as close as possible to the MI line, posterior side) in all cases. The length of the MI line was measured with the 3D mapping system. The maximal amplitude of the bipolar EGMs at 3 different positions on the MI was also measured in each patient in SR before the start of MI ablation.

Patients were randomized in a 1:1 fashion to 1 of $2 \mathrm{MI}$ ablation arms (Figs. 1 and 2).

(1) Posterior MI ablation (group 1): The linear lesion was performed from the posterior mitral annulus to the left inferior PV (posterior side) by drawing a vertical line. Briefly, the ablation catheter (Navistar ${ }^{\circledR}$ Thermocool ${ }^{\circledR}$ $3.5 \mathrm{~mm}$; Biosense Webster) was introduced through a long sheath (SL0, St. Jude Medical, Minneapolis, MN, USA) to aid stability during ablation. Using a clockface analogy, ablation commenced at 6 o'clock (left anterior oblique [LAO] view) on the ventricular edge of the mitral annulus. The catheter was dragged gradually back into the sheath to extend the lesion along the isthmus in a linear fashion up to the left inferior PV ostium. Every effort was a made to perform a truly linear ablation and to keep the point-by-point ablation line as narrow as possible not to interfere with the anterolateral MI area whose ablation was tested in group 2.

(2) Anterolateral MI ablation (group 2): The linear lesion was performed from the anterolateral mitral annulus to the left inferior PV (anterior side) by drawing a horizontal line. In this case, ablation commenced at 3 o'clock (LAO view) on the ventricular edge of the lateral mitral annulus. The catheter was dragged gradually back into the sheath to extend the lesion along the isthmus in a linear fashion up to the anterior part of the left inferior PV ostium.

In both groups, after initial ablation, endocardial and epicardial mapping was performed to identify and ablate any gaps. Endocardial ablation was performed with a flow rate of 12-60 $\mathrm{mL} / \mathrm{min}$, and maximal temperature of $45^{\circ} \mathrm{C}$ (usually below $42^{\circ} \mathrm{C}$ ) with power set at $35 \mathrm{~W}$. Epicardial ablation was performed in the coronary sinus (CS) with power limited to $25 \mathrm{~W}$. The endpoint of ablation was achievement of bidirectional MI conduction block demonstrated using previously described techniques and criteria. ${ }^{14,15}$

MI ablation was stopped after a maximum of 30 minutes of RF application, and this was considered as failure. 


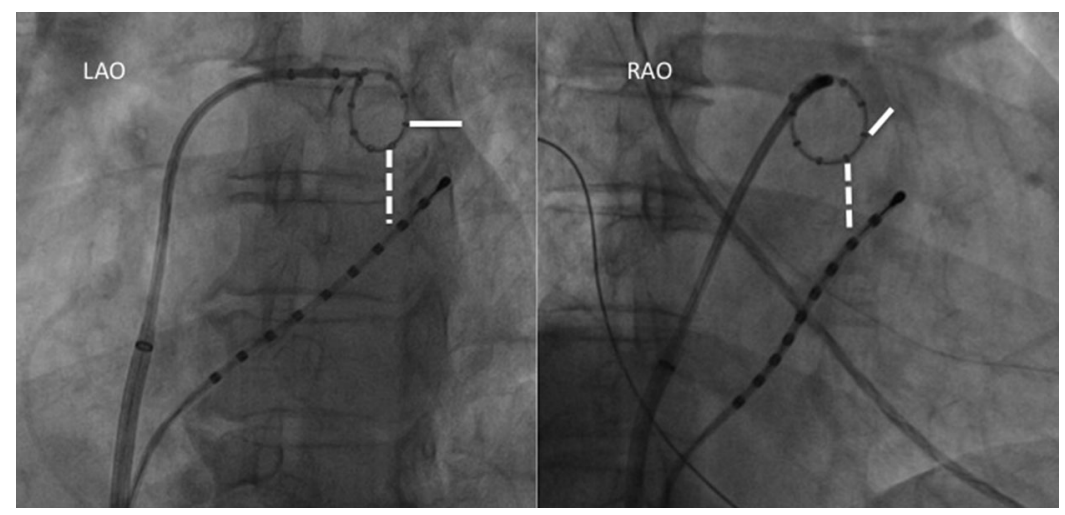

Figure 1. $L A O$ and RAO views of the heart displaying a decapolar catheter in the coronary sinus and a Lasso catheter in the left inferior pulmonary vein. The dashed line illustrates the posteror mitral isthmus line, whereas the full line displays the anterolateral mitral isthmus line.

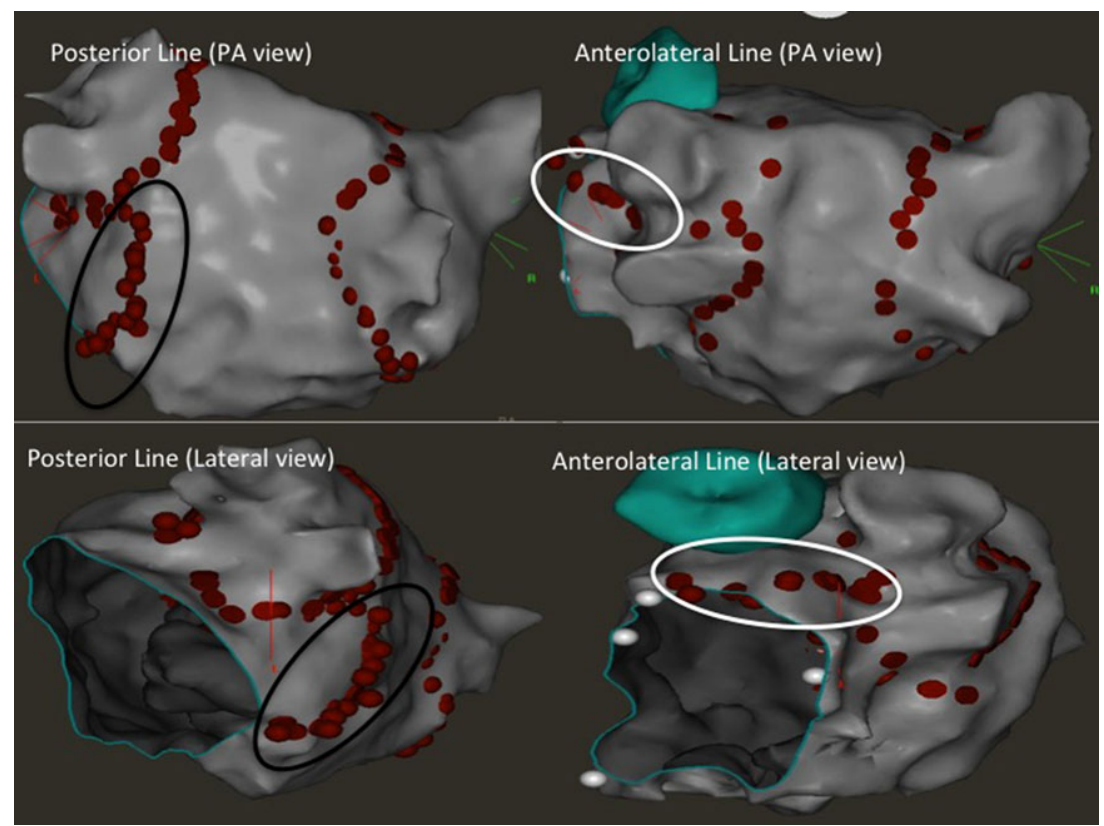

Figure 2. Electroanatomic maps and ablation lesions in patients with a posterior MI line (group 1) and an anterolateral MI line (group 2). The relevant lesion sets are highlighted with a black ellipse (posterior MI line) and a white ellipse (anterior line). A and B: A modified posterior view of the left atrium: $C$ and D: A modified left lateral view. For a high quality, full color version of this figure, please see Journal of Cardiovascular Electrophysiology's website: www.wileyonlinelibrary.com/journal/jce

\section{Statistical Analysis}

Continuous variables were presented as mean $\pm \mathrm{SD}$, or median (and interquartile range [IQR]), depending on normality of distribution. Categorical variables are presented as percentages (\%) and counts. Continuous variables were compared with Student $t$-test or the Wilcoxon rank-sum test (nonnormally distributed data), and frequencies with chisquare analysis or Fisher's exact test for small numbers, as appropriate. Univariate logistic regression was performed to estimate the magnitude of risk associated with potential risk factors for MI block. Where appropriate, the receiveroperator characteristic (ROC) curve was determined to evaluate the performance of the best independent predictor of MI block. The optimal cut-off point was chosen as the combination with the highest sensitivity and specificity (Youden Index).

For the stepwise logistic regression analysis, the continuous variables were appropriately transformed where required to binary variables based on the ROC curve analysis. A multivariable Cox proportional hazard model was used to investigate the association between baseline characteristics and procedural data (inclusion of all parameters showing a $\mathrm{P}<0.05$ at univariate analysis) and MI block. For each variable, odds ratio $(\mathrm{OR}), 95 \%$ confidence interval $(\mathrm{CI})$, and Wald test $\mathrm{P}$ values of the final model were displayed. All tests of significance were 2-tailed, and a $\mathrm{P}$ value of less than 0.05 was considered to indicate statistical significance. All analyses were performed with SPSS 18.0.

\section{Results}

\section{Clinical Characteristics}

Patients' characteristics are shown in Table 1 . Of the 40 patients enrolled in the study, 19 patients were randomized to group 1 and 21 patients were randomized to group 2 . There was no significant difference in the age, gender, type of AF, presence of structural heart disease, duration of uninterrupted persistent $\mathrm{AF}$, use of antiarrhythmic drugs prior to ablation, and echocardiographic left heart dimensions between the 2 arms.

\section{Ablation}

MI block was achieved in 14/19 patients (74\%) in group 1 and in $15 / 21$ patients $(71 \%)$ in group $2(\mathrm{P}=\mathrm{NS})$. Total MI radiofrequency time $(18 \pm 7 \mathrm{~min}$ vs. $17 \pm 8 \mathrm{~min} ; \mathrm{P}=\mathrm{NS})$ and ablation rate in the CS $(81 \%$ vs. $77 \% ; \mathrm{P}=\mathrm{NS})$ were similar between groups (Table 2). No complication occurred during the procedures. 


\section{TABLE 1}

Baseline Characteristics ( $\mathrm{n}=40$ patients)

Female gender

Age (years)

History of AF (months)

Continuous AF duration (months)

Long standing persistent $\mathrm{AF}$

Obesity (BMI > 30)

Amiodarone at time of procedure

Left atrial diameter $(\mathrm{mm})$

Left ventricular ejection fraction (\%)

Hypertension

Structural heart disease

$\mathrm{CHADS}_{2}$ score $\geq 2$

Values are given as $\mathrm{n}(\%)$ or as mean $\mathrm{SD}$, unless otherwise noted. $\mathrm{AF}=$ atrial fibrillation; $\mathrm{BMI}=$ body mass index.

\section{TABLE 2}

Univariate Analysis Comparing MI Ablation Groups

\begin{tabular}{lccc}
\hline Variable & $\begin{array}{c}\text { Posterior } \\
\text { MI Line } \\
(\mathbf{n}=\mathbf{1 9})\end{array}$ & $\begin{array}{c}\text { Anterolateral } \\
\text { MI Line } \\
(\mathbf{n = 2 1})\end{array}$ & P Value \\
\hline Bidirectional MI block & $14(74 \%)$ & $15(71 \%)$ & 0.87 \\
Length of MI line (mm) & $27 \pm 7$ & $28 \pm 8$ & 0.73 \\
Mean MI voltage (mV) & $1.16 \pm 0.74$ & $1.33 \pm 0.80$ & 0.50 \\
Fluoroscopy time (minutes) & $14 \pm 7$ & $15 \pm 9$ & 0.90 \\
Total RF time (minutes) & $23 \pm 8$ & $25 \pm 11$ & 0.59 \\
RF time in CS (minutes) & $4 \pm 3$ & $4 \pm 5$ & 0.93 \\
RF in CS & $16(84 \%)$ & $18(86 \%)$ & 0.89 \\
Delay across MI line (milliseconds) & $131 \pm 40$ & $139 \pm 36$ & 0.46 \\
\hline
\end{tabular}

$\mathrm{MI}=$ mitral isthmus; $\mathrm{RF}=$ radiofrequency; $\mathrm{CS}=$ coronary sinus.

TABLE 3

Univariate Analysis Comparing the Successful MI Ablation Group with the MI Ablation Failure Group

\begin{tabular}{lccc}
\hline Variable & $\begin{array}{c}\text { Success } \\
(\mathbf{n = 2 9 )}\end{array}$ & $\begin{array}{c}\text { Failure } \\
(\mathbf{n}=\mathbf{1 1})\end{array}$ & P Value \\
\hline Age (years) & $52 \pm 7$ & $55 \pm 10$ & 0.35 \\
History of AF (months) & $51 \pm 43$ & $52 \pm 39$ & 0.94 \\
Cont. AF duration (months) & $10 \pm 10$ & $19 \pm 17$ & $0.04^{*}$ \\
Obesity (BMI >30) & $7(25 \%)$ & $7(64 \%)$ & $0.03^{*}$ \\
Amiodarone at time of procedure & $11(37 \%)$ & $2(18 \%)$ & 0.29 \\
LA diameter (mm) & $45 \pm 4$ & $48 \pm 6$ & 0.07 \\
LV ejection fraction (\%) & $56 \pm 15$ & $54 \pm 12$ & 0.75 \\
Hypertension & $11(37 \%)$ & $4(36 \%)$ & 0.93 \\
Structural heart disease & $10(38 \%)$ & $5(45 \%)$ & 0.52 \\
Length of MI line (mm) & $24 \pm 5$ & $34 \pm 6$ & $<0.001^{*}$ \\
Mean MI voltage (mV) & $1.05 \pm 0.69$ & $1.76 \pm 0.75$ & $<0.01^{*}$ \\
RF in CS & $23(79 \%)$ & $11(100 \%)$ & 0.10 \\
\hline
\end{tabular}

Abbreviations as for Tables 1 and 2.

\section{Line Characteristics}

Overall, patients with incomplete MI block had a longer MI line length (34 $\pm 6 \mathrm{~mm}$ vs. $24 \pm 5 \mathrm{~mm}$; $\mathrm{P}<0.001)$, a higher mean bipolar voltage along the MI line (1.75 \pm $0.74 \mathrm{mV}$ vs. $1.05 \pm 0.69 \mathrm{mV} ; \mathrm{P}<0.01)$, a longer history of continuous AF $(19 \pm 17$ months vs. $10 \pm 10$ months; $\mathrm{P}<0.05)$, and a higher rate of obesity $(75 \%$ vs. $16 \%$; $\mathrm{P}<0.05$ ) (Table 3).

In multivariate analysis, a decreased length of the MI line was an independent risk factor for success in blocking the MI (OR 1.5; 95\% CI 1.1-2.1; P < 0.05) after adjustment for mean MI voltage $<1.17 \mathrm{mV}$, absence of obesity, and absence of longstanding persistent AF.

Interestingly, we found a moderate correlation between the length of the mitral line and the ablation time (correlation coefficient $=0.575 ; \mathrm{P}<0.001$ ).

\section{Discussion}

This study highlights the importance of the MI length for achieving bidirectional MI electrical block. It also confirms the difficulty in achieving conduction block across the MI line that still represents the most difficult step (when necessary) during AF ablation.

\section{Difficulty in MI Abation}

MI line still represents the most difficult step in AF ablation. Reported success rates range from $65 \%$ to $92 \%{ }^{5,6,8,9}$ Increased success rates often require higher power for ablation and this is associated with an increased risk of tamponade. The MI is a corridor of uncertainty and the technical challenge when performing an MI line can be explained by (1) the wall thickness of the MI, (2) the presence of numerous recesses and cavities along the MI, ${ }^{16}(3)$ the presence of epicardial muscular connections necessitating ablation within the CS in the majority of patients, ${ }^{5}$ (4) the anatomical location with the presence of the LA appendage, and (5) the technical challenge of catheter stability as compared to the CTI line for instance.

Postmortem anatomical and in vivo studies have shown that the atrial myocardium from the left inferior pulmonary vein to the lateral aspect of the mitral valve has a wall thickness up to $10 \mathrm{~mm} .{ }^{17}$

Our study confirms the technical challenge of MI ablation, with a global success rate of only $73 \%$. We have to notice that power has been restricted to less than 35 Watts with the intention to avoid serious complication, which has been the case. For the purpose of the study, we have also limited the duration of RF application for the MI line to less than 30 minutes, even if conduction delay had significantly increased.

This difficulty in achieving conduction block at the MI line raised the question of the timing and overall necessity to perform this linear lesion. The global aim of linear lesions is to reproduce the surgical MAZE procedure, based on the concept of macroreentrant loop and wandering wavelets participating in the AF process. Several studies have described the additional benefit of the LMI line particularly for persistent $\mathrm{AF}^{6,16}$ but at the expense of more complications. Importantly, even when not performed during AF, MI line has frequently to be done after $\mathrm{AF}$ termination for subsequent peri-mitral flutter. ${ }^{6}$

\section{Localization of the MI Line}

We investigated the potential benefit of MI localization in the success rate of this technique. Theoretically, a more posterior approach allows for avoiding the area of the LA appendage, which can be a source of perforation and is probably the thickest part of the MI area. However, posterior application always represents a potential risk for atrio-esophageal fistula. Our study did not evidence any difference in performance concerning the localization. During multivariate 
analysis, only the length of the MI line was important for procedural success.

Interestingly, a higher bipolar voltage along the MI line was not associated with more failure of bidirectional block in multivariate analysis. During CTI ablation, ablation targeting maximum voltage has been shown to reduce RF duration, ${ }^{18}$ suggesting the presence of discrete muscle bundles separated by connective tissue. ${ }^{19}$ This seems not to be the case for the MI where most electrograms were relatively ample and with no difference in the reported success rate.

Interestingly, Pak et al. ${ }^{20}$ studied an alternative approach to the classic left anterolateral or posterior MI, namely a line on the LA anterior wall. The length of this anterior line during ablation was longer $(37.9 \pm 3.4 \mathrm{~mm}$ vs. $26.6 \pm 3.2 \mathrm{~mm}, \mathrm{P}<$ 0.0001 ), but achievement of bidirectional block was higher $(68 \%$ vs. $32 \%, \mathrm{P}<0.0001)$ than with a left lateral isthmus ablation. In our own experience, we also found the left lateral isthmus to be shorter and the achievement of bidirectional block less challenging to achieve than when ablating the left anterior line between the anterior MI and the roof line or the right superior pulmonary vein.

\section{Clinical Implications}

This study would suggest that there might be utility in measuring the shortest way from the mitral annulus to the left inferior PV. This could potentially increase the success rate of MI line ablation, when this line is necessary either during $\mathrm{AF}$ of for the occurrence of a perimitral flutter. However, other parameters can be taken into account: the stability of the catheter during ablation, access to the distal CS as this is necessary in most of the cases, the presence of the esophagus near the left veins or a close position of the LA appendage as compared to the left inferior PV.

\section{Limitations}

This is a single-center study with limited patient numbers. No CT/MR imaging and image integration was performed, which may have increased the procedural success. Anatomical differences of the MI in cases that were successful versus those that were unsuccessful were also not evaluated. However, all of the procedures have been performed with a 3D electro-anatomical system. At the time of our study, we did not routinely use force sensing catheters and therefore we can provide no information on the force integral necessary to achieve MI block.

Finally, no steerable sheath was used, which has been demonstrated to increase the success rate.

\section{Conclusions}

In patients undergoing persistent $\mathrm{AF}$ ablation using the stepwise approach, decreased length but not anatomical location of the MI line as assessed with a 3D mapping system predicts success in achieving bidirectional mitral isthmus block.

\section{References}

1. Cheema A, Dong J, Dalal D, Marine JE, Henrikson CA, Spragg D, Cheng A, Nazarian S, Bilchick KC, Almasry I, Sinha S, Scherr D, Halperin H, Berger R, Calkins H: Circumferential ablation with pulmonary vein isolation in permanent atrial fibrillation. Am J Cardiol 2007; $99: 1425-1428$.

2. Elayi CS, Verma A, Di Biase L, Ching CK, Patel D, Barrett C, Martin D, Rong B, Fahmy TS, Khaykin Y, Hongo R, Hao S, Pelargonio G, Dello Russo A, Casella M, Santarelli P, Potenza D, Fanelli R, Massaro R, Arruda M, Schweikert RA, Natale A: Ablation for longstanding permanent atrial fibrillation: Results from a randomized study comparing three different strategies. Heart Rhythm 2008;5:1658-1664.

3. Tilz RR, Rillig A, Thum AM, Arya A, Wohlmuth P, Metzner A, Mathew S, Yoshiga Y, Wissner E, Kuck KH, Ouyang F: Catheter ablation of long-standing persistent atrial fibrillation: 5-year outcomes of the Hamburg Sequential Ablation Strategy. J Am Coll Cardiol 2012;60:1921-1929.

4. O'Neill MD, Wright M, Knecht S, Jais P, Hocini M, Takahashi Y, Jonsson A, Sacher F, Matsuo S, Lim KT, Arantes L, Derval N, Lellouche N, Nault I, Bordachar P, Clementy J, Haissaguerre M: Longterm follow-up of persistent atrial fibrillation ablation using termination as a procedural endpoint. Eur Heart J 2009;30:1105-1112.

5. Jais P, Hocini M, Hsu LF, Sanders P, Scavee C, Weerasooriya R, Macle L, Raybaud F, Garrigue S, Shah DC, Le Metayer P, Clementy J, Haissaguerre M: Technique and results of linear ablation at the mitral isthmus. Circulation 2004:110:2996-3002.

6. Knecht S, Hocini M, Wright M, Lellouche N, O’Neill MD, Matsuo S, Nault I, Chauhan VS, Makati KJ, Bevilacqua M, Lim KT, Sacher F, Deplagne A, Derval N, Bordachar P, Jais P, Clementy J, Haissaguerre $\mathrm{M}$ : Left atrial linear lesions are required for successful treatment of persistent atrial fibrillation. Eur Heart J 2008;29:2359-2366.

7. Parkash R, Tang AS, Sapp JL, Wells G: Approach to the catheter ablation technique of paroxysmal and persistent atrial fibrillation: A meta-analysis of the randomized controlled trials. J Cardiovasc Electrophysiol 2011;22:729-738.

8. Matsuo S, Yamane T, Date T, Hioki M, Narui R, Ito K, Tanigawa S, Nakane T, Yamashita S, Tokuda M, Inada K, Nojiri A, Kawai M, Sugimoto K, Yoshimura M: Completion of mitral isthmus ablation using a steerable sheath: Prospective randomized comparison with a nonsteerable sheath. J Cardiovasc Electrophysiol 2011;22:1331-1338.

9. Miyazaki S, Shah AJ, Liu X, Jadidi AS, Nault I, Wright M, Forclaz A, Linton N, Xhaet O, Rivard L, Derval N, Knecht S, Sacher F, Hocini M, Jais P, Haissaguerre M: Preprocedural clinical parameters determining perimitral conduction time during mitral isthmus line ablation. Circ Arrhythm Electrophysiol 2011;4:287-294.

10. Wong KC, Betts TR: A review of mitral isthmus ablation. Indian Pacing Electrophysiol J 2012;12:152-170.

11. Rostock T, Salukhe TV, Steven D, Drewitz I, Hoffmann BA, Bock K, Servatius H, Mullerleile K, Sultan A, Gosau N, Meinertz T, Wegscheider K, Willems S: Long-term single- and multiple-procedure outcome and predictors of success after catheter ablation for persistent atrial fibrillation. Heart Rhythm 2011;8:1391-1397.

12. Sawhney N, Anand K, Robertson CE, Wurdeman T, Anousheh R, Feld GK: Recovery of mitral isthmus conduction leads to the development of macro-reentrant tachycardia after left atrial linear ablation for atrial fibrillation. Circ Arrhythm Electrophysiol 2011;4:832-837.

13. Calkins H, Kuck KH, Cappato R, Brugada J, Camm AJ, Chen SA, Crijns HJ, Damiano RJ Jr, Davies DW, DiMarco J, Edgerton J, Ellenbogen K, Ezekowitz MD, Haines DE, Haissaguerre M, Hindricks G, Iesaka Y, Jackman W, Jalife J, Jais P, Kalman J, Keane D, Kim YH, Kirchhof P, Klein G, Kottkamp H, Kumagai K, Lindsay BD, Mansour M, Marchlinski FE, McCarthy PM, Mont JL, Morady F, Nademanee K, Nakagawa H, Natale A, Nattel S, Packer DL, Pappone C, Prystowsky E, Raviele A, Reddy V, Ruskin JN, Shemin RJ, Tsao HM, Wilber D: 2012 HRS/EHRA/ECAS Expert Consensus Statement on Catheter and Surgical Ablation of Atrial Fibrillation: Recommendations for patient selection, procedural techniques, patient management and follow-up, definitions, endpoints, and research trial design. Europace 2012;14:528-606.

14. Jais P, Hocini M, O'Neill MD, Klein GJ, Knecht S, Sheiiro M, Arentes L, Kodali S, Clementy J, Haissaguerre M: How to perform linear lesions. Heart Rhythm 2007;4:803-809.

15. Shah D, Haissaguerre M, Takahashi A, Jais P, Hocini M, Clementy J: Differential pacing for distinguishing block from persistent conduction through an ablation line. Circulation 2000;102:1517-1522.

16. Fassini G, Riva S, Chiodelli R, Trevisi N, Berti M, Carbucicchio C, Maccabelli G, Giraldi F, Bella PD: Left mitral isthmus ablation associated with PV Isolation: Long-term results of a prospective randomized study. J Cardiovasc Electrophysiol 2005;16:1150-1156. 
17. Becker AE: Left atrial isthmus: Anatomic aspects relevant for linear catheter ablation procedures in humans. J Cardiovasc Electrophysiol 2004; 15:809-812.

18. Gula LJ, Redfearn DP, Veenhuyzen GD, Krahn AD, Yee R, Klein GJ, Skanes AC: Reduction in atrial flutter ablation time by targeting maximum voltage: Results of a prospective randomized clinical trial. J Cardiovasc Electrophysiol 2009;20:1108-1112.
19. Waki K, Saito T, Becker AE: Right atrial flutter isthmus revisited: Normal anatomy favors nonuniform anisotropic conduction. J Cardiovasc Electrophysiol 2000;11:90-94.

20. Pak HN, Oh YS, Lim HE, Kim YH, Hwang C: Comparison of voltage map-guided left atrial anterior wall ablation versus left lateral mitral isthmus ablation in patients with persistent atrial fibrillation. Heart Rhythm 2011;8:199-206. 